\title{
Interactive effects between auditory and vibrotactile stimuli
}

\author{
KAL M. TELAGE and JANET C. SCOTT \\ Department of Speech Pathology and Audiology, School of Allied Health Professions \\ Ithaca College, Ithaca, New York 14850
}

\begin{abstract}
Sensory effects between auditory and vibrotactile stimuli were investigated by obtaining lingual tactile thresholds under four conditions including no masking, 70, 90, and $110 \mathrm{~dB}$ SPL of wide-band noise. Obtained thresholds were highly similar, indicating no shifts in tactile sensitivity from auditory interactions. Findings suggest that dorsal lingual mechanoreceptors may function as independent afferent mechanisms.
\end{abstract}

The interaction between auditory and oral sensory feedback mechanisms reported by Sussman (1970) has generated a series of studies that have attempted to alter lingual tactile sensitivity through some form of auditory interference. Fucci, Crary, Warren, and Bond (1977) obtained poorer posttest lingual vibrotactile thresholds for experimental subjects after reading a 3-min passage while being exposed to $80 \mathrm{~dB}$ HTL of wide-band noise. Fucci et al. concluded that the auditory masking produced a subsequent threshold shift in tongue sensitivity to vibrotactile stimuli. Crary, Fucci, and Bond (1979) investigated lingual threshold sensitivity by exposing subjects to $90 \mathrm{~dB}$ SPL of babble noise while they read prose passages for intervals of 30 to $80 \mathrm{sec}$. The investigators reported that exposure to noise resulted in subsequently poorer vibrotactile threshold, which in their view indicated sensory feedback interactions. Telage and Scott (1980), using a similar paradigm, presented noise exposure levels at $90 \mathrm{~dB}$ SPL to experimental subjects but did not find significant differences between the pretest and posttest virbrotactile thresholds of the experimental and control groups. Telage and Scott explained the discrepancy between their work and previous studies as possibly due to differences in test procedures. Telage and Scott (1980) did not use a clamping procedure that compresses lingual tissues and forces more ventrally based Pacinian corpuscles to the surface. It has previously been shown that the dorsal lingual receptors differ in response characteristics and may be stimulated independently if lingual structures are not distorted (Telage \& Petrosino, 1978). Telage and Scott (1980) concluded that the two classes of lingual mechanoreceptors project sensory patterns that interact differently with auditory phenomena.

Recently, Petrosino, Fucci, and Lowe (1981) investigated the Lombard effect for lingual tactile thresholds. They concluded that neither vocal intensity nor auditory masking produced significant posttest threshold shifts in lingual vibrotactile sensitivity. It is interesting to note that this study also utilized a methodology that did not clamp the tongue.

A review of the experimental designs of the reported studies shows that auditory interference always preceded lingual tactile sensitivity measures. The assumption has been that possible shifts in vibrotactile thresholds would persist long enough to be observed during posttesting. Since this may not be the case, it is clear that none of these studies has ruled out the sensory interaction hypothesis. The present investigation attempted to simultaneously assess interactions between auditory and oral sensory feedback channels.

\section{METHOD}

\section{Subjects}

Twenty-four adult subjects with normal speech and hearing and no known sensory or motor impairments served as subjects. Lingual vibrotactile thresholds were determined for each subject using four simultaneous conditions: (1) no masking, (2) 70-dBSPL masking, (3) 90-dB-SPL masking, and (4) 110-dB-SPL masking. The order of presentation of conditions was counterbalanced so that no two subjects received the same sequence.

\section{Apparatus}

Figure 1 presents a block diagram of the instrument package used in this study. The stimulus control unit is composed of

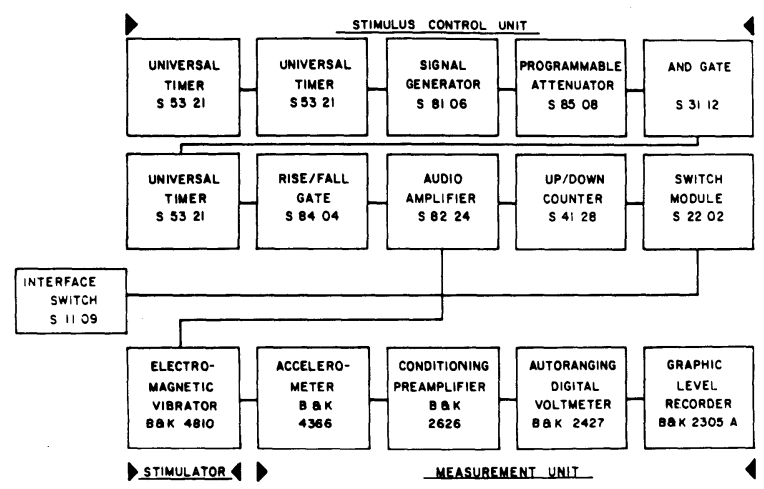

Figure 1. A block diagram of the automated instrumentation system. 


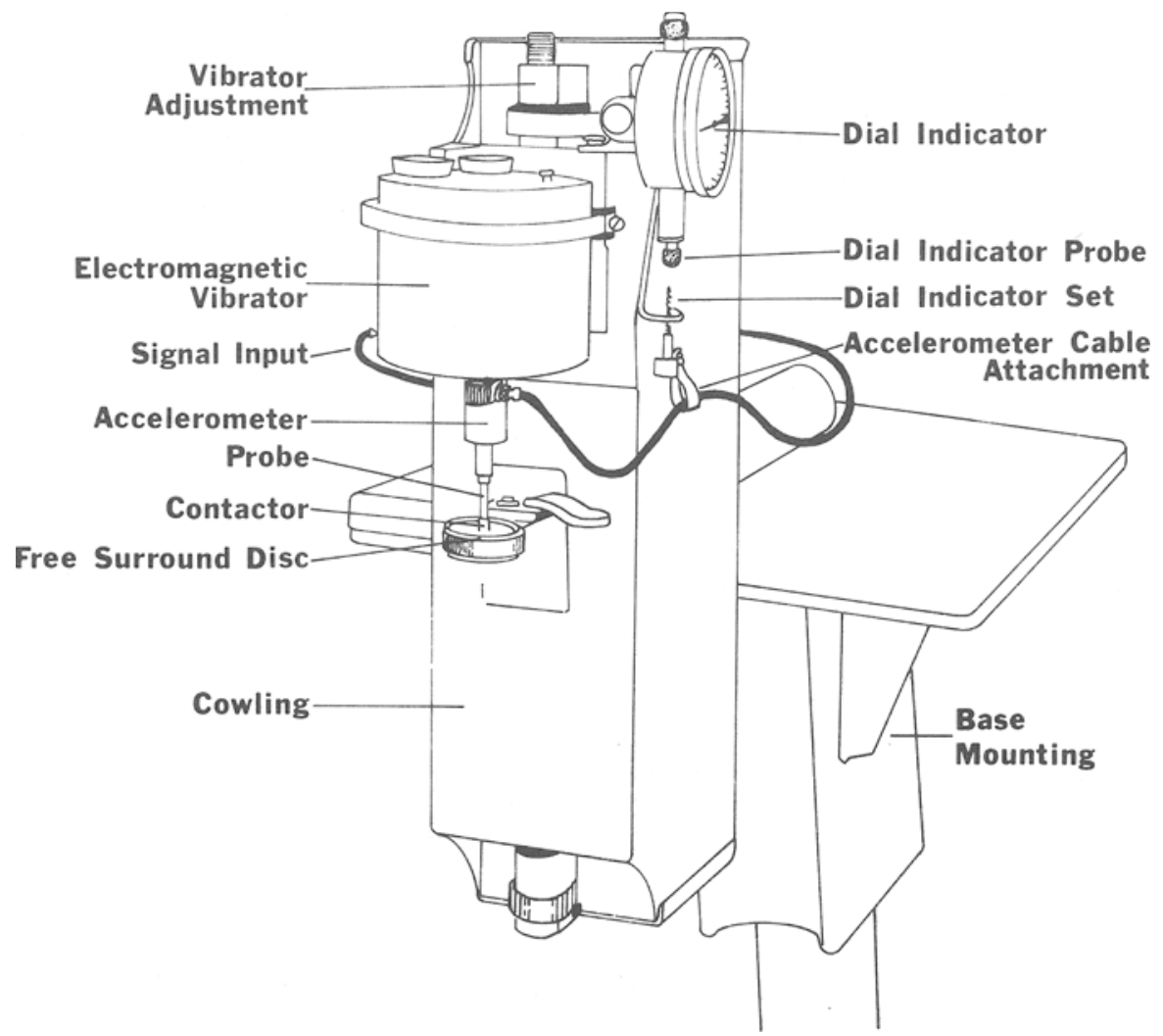

Figure 2. A schematic diagram of the oral vibrotactile stimulator.

Coulbourn solid state logic modules. These units generate pulsed vibratory signals that may be varied in frequency, intensity, and temporal characteristics. Three universal times are programmed to control signal duration and duty cycle. The timers gate a selectable rise-fall module on and off. This continuously adjustable electronic switch was set to generate a risefall time of $100 \mathrm{msec}$. The signal from the rise-fall module was fed into a precision signal generator and an audio mixer amplifier. Stimulus intensities were varied in increments of 256 $.5-\mathrm{dB}$ steps by passing them through a programmable attenuator and an 8-bit binary up-down counter. Pulsed signals from the stimulus control unit drive the electromagnetic minivibrator that is the stimulus-producing aggregate of the system.

Figure 2 presents a schematic diagram of the vibrator and modified clamp assembly portion of the vibrotactile stimulator. The lower clamping arm and its disk were removed. The upper clamping arm with a free-surround disk was retained. All contact points between the vibrator and cowling were insulated by rubber stripping.

The vibrator probe with attached contactor was lowered $1 \mathrm{~mm}$ below the lower surface of the free-surround disk. The area of the contactor was $.128 \mathrm{~cm}^{2}$. The diameter of the opening in the free-surround disk was $2 \mathrm{~mm}$ larger than the diameter of the contactor.

An accelerometer mounted on the probe measured contactor displacement as a small voltage. Displacement voltages were directly amplified by a conditioning preamplifier that was set to emit $100 \mathrm{mV} / \mathrm{g}$ of acceleration. Signals were constantly monitored by an autoranging digital voltmeter set to read peak displacement values in millivolts.

A wide-band noise generator presented auditory masking at 70,90 , and $100 \mathrm{~dB}$ SPL through TDH-39 headphones. A detailed explanation of the instrumentation system is provided by Telage (1979).

\section{Procedure}

All subjects received an orientation to the instrumentation and procedures prior to actual testing. In addition to the explanation, each subject experienced the experimental process and was required to provide consistent lingual tactile thresholds to a $250-\mathrm{Hz}$ stimulus within a specified range of sensitivity. All subjects used in the experiment met this pretest threshold criteria.

During testing, subjects were comfortably seated in an adjustable chair and were asked to place the upper lingual surface against a free-surround disk. Thresholds for lingual tactile sensitivity at a frequency of $250 \mathrm{~Hz}$ were obtained using an ascending psychophysical method of limits. Subjects raised their fingers to signal detection of the stimulus on the tongue. The median of three test trials was accepted as each subject's individual threshold. Data, initially recorded in millivolts, were subsequently converted to microns of peak displacement using an acceleration formula (Telage, 1979).

\section{RESULTS AND DISCUSSION}

Table 1 presents means and standard deviations expressed in microns of peak displacement for each experimental condition. Mean values between each set of conditions were nonsignificant, indicating no interactive effects between auditory masking and threshold responsivity. 
Table 1

Means and Standard Deviations for Lingual Vibrotactile Thresholds

\begin{tabular}{ccc}
\hline & \multicolumn{2}{c}{$\begin{array}{c}\text { Data in Microns of } \\
\text { Peak Displacement }\end{array}$} \\
\cline { 2 - 3 } Condition & Mean & SD \\
\hline No Masking & 1.85 & .42 \\
$70 \mathrm{~dB}$ SPL & 1.79 & .41 \\
$90 \mathrm{~dB}$ SPL & 1.76 & .41 \\
$110 \mathrm{~dB}$ SPL & 1.76 & .53 \\
\hline
\end{tabular}

Note-All SDs were nonsignificant.

It is interesting to speculate about this finding and earlier studies by Crary et al. (1979) and Fucci et al. (1977). An important difference in methodologies concerns clamping or not clamping the lingual structures while determining threshold sensitivity. This study, a previous study (Telage \& Scott, 1980), and a recent investigation by Petrosino et al. (1981) altered lingual positioning procedures so as not to compress together the tongue's ventral and dorsal tissues. This experimental modification better ensures the stimulation of surface transducers that respond to light pressure. It is reasonable to assume that studies that compressed the tongue's tissues increase the possibility of exciting more ventrally lying Pacinian corpuscles, which are responsive to deep pressure.

This difference in methodologies clearly shows results in which masking interfered with threshold sensitivity (lingual compression studies) and findings in which sensitivity was unaffected by noise exposure (noncompression studies). It is possible to explain these differences in terms of the duplex theory of mechanoreception postulated by Verrillo (1966), who advocated two distinct receptor mechanisms for transduction of pressure sensitivity from the tongue. The dorsal surface system does not summate in projecting to higher levels in the CNS. The more ventral, deeper lying Pacinian corpuscle system is thought to summate energy both spacially and temporally in a manner quite similar to the auditory sensory system (Zwislocki, 1960).

It is reasonable to theorize that the Pacinian transduction system works in concert with audition to augment a balanced sensory input for complex motor synergies, particularly those used for speech (Sussman, 1970). If this is the case, experiments that have clamped the tongue may have excited this receptor population. Subsequent interactions between tactile input and auditory masking afferents could have resulted in observed vibrotactile threshold shifts.

This supposition does not conflict with Sussman's (1970) view of balanced auditory-tactile interactions. Rather, it provides support for the nature of tactile transducers that may act in synchrony with the auditory system during complex oral motor synergies. Other research findings argue that mechanoreceptors located at the dorsal surface of the tongue constitute a more independent afferent mechanism (Telage \& Petrosino, 1978). Further research is needed to better identify the functional nature of either system for the control of motor events.

\section{REFERENCES}

Crary, M. A., Fucci, D., \& Bond, Z. Timed auditory interruption during speech: Oral sensory and temporal articulatory changes. Journal of Phonetics, 1979, 7, 335-342.

Fucci, D., Crary, M. A., Warren, J., \& Bond, Z. S. An interaction between auditory and oral sensory feedback in speech regulation. Perceptual and Motor Skills, 1977, 45, 123-129.

Petrosino, L., Fucci, D., \& Lowe, R. Role of the Lombard effect in lingual vibrotactile thresholds. Perceptual and Motor Skills, 1981, 52, 263-268.

Sussman, H. The role of sensory feedback in tongue movement control. Journal of Auditory Research, 1970, 10, 296-321.

Telage, K. Automated instrumentation for research on the lingual tactile sensory system. Review of Scientific Instruments, 1979, 50, 1296-1298.

Telage, K., \& Petrosino, L. Testing procedures for measuring oral vibrotactile thresholds: III. Effects obtained using a nonclamping method. Bulletin of the Psychonomic Society, 1978, 12, 291-295.

TelaGe, K., \& ScotT, J. C. Effects of auditory interference upon observed lingual tactile thresholds. Bulletin of the Psychonomic Society, 1980, 15, 422-424.

Verrillo, R. T. Specificity of a cutaneous receptor. Perception \& Psychophysics, 1966, 1, 149-153.

Zwislocki, J. A theory of temporal summation. Journal of the Acoustical Society of America, 1960, 32, 1046-1060.

(Received for publication December 7, 1981.) 